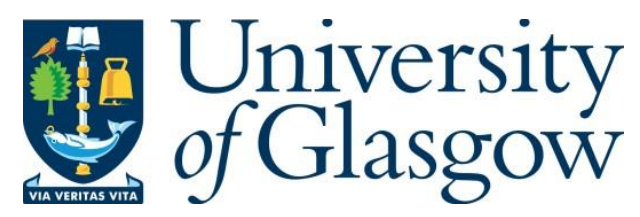

Heidari, H., Liu, F. and Dahiya, R. (2017) Towards flexible magnetoelectronics for robotic applications. In: 2017 2nd Asia-Pacific Conference on Intelligent Robot Systems (ACIRS), Wuhan, China, 16-18 Jun 2017, pp. 295-298. ISBN 9781509067923.

There may be differences between this version and the published version. You are advised to consult the publisher's version if you wish to cite from it.

http://eprints.gla.ac.uk/144631/

Deposited on: 08 August 2017

Enlighten - Research publications by members of the University of Glasgow http://eprints.gla.ac.uk 


\title{
Towards Flexible Magnetoelectronics for Robotic Applications
}

\author{
Hadi Heidari, Fengyuan Liu, Ravinder Dahiya \\ Bendable Electronics and Sensing Technologies (BEST) group, School of Engineering, University of Glasgow, G12 8QQ, UK \\ Hadi.Heidari@glasgow.ac.uk, Ravinder.Dahiya@glasgow.ac.uk
}

\begin{abstract}
This paper presents the technological advancements in the field of flexible magnetic sensors for robotics applications. Various magnetic devices (e.g. Hall, GMR, AMR and TMR) have been studied and their suitability for flexible application has been presented. Further, the system level integration of magnetic sensors in robotics is briefly discussed. With rapid development in flexible electronics, a robot with multi-functional conformable electronic skin will be possible in the foreseeable future. This will also open new avenues for a wide range of other applications including wearable electronics and interactive electronic-skin for robots and prosthesis.
\end{abstract}

Keywords-Flexible Electronics, Magnetic Sensors, Hall Effect, Magnetoresistance, AMR, GMR, TMR.

\section{INTRODUCTION}

The integration of magnetic elements into electronic devices, often referred to as Magnetoelectronics [1-4], is an interesting development that has caught the attention of many working in the field of flexible electronics [5-9]. Magnetic sensors including Hall effect sensor, giant magnetoresistance (GMR) sensor, anisotropic magnetoresistance sensor (AMR) sensor, tunnel magnetoresistance (TMR) sensor etc., has been studied intensively during the past few years. The ability of sensing external magnetic intensity has been applied in many fields for application such as contactless switching, magnetic anomaly detection and medical/biological application [10]. Variety of magnetic sensors have also been used in robotics and autonomous systems for tasks such as position monitoring [11], human activity recognition and estimation [12, 13], flight control of biomimetic robot [14], inspection of the energy consumption [15] and error correction in teleoperation system [16] etc.

Studied for past several decades, the magnetic sensors are generally rigid and stiff. For example, the most commonly used Hall sensor, fabricated by CMOS technology, is around $400 \mu \mathrm{m}$ thick and not flexible. However, the curved surfaces of robotic body demand the electronics and sensors to be on flexible and conformable surfaces. The flexibility, and sometimes stretchability, of a devices and circuits is favourable as it makes the movement easy (Fig. 1). With the development of material science and nanotechnology, different types of flexible magnetic sensors have been demonstrated recently.

Next generation wearable electronic systems will require ultra-thin and flexible devices, body tracking, and relative position monitoring systems. Such devices will fulfil the needs of robotics, functional medical implants, and imperceptible and wearable electronics. Magnetic field sensors offer the possibility to sense and respond to external magnetic fields, which when integrated into electronic skin will bring new

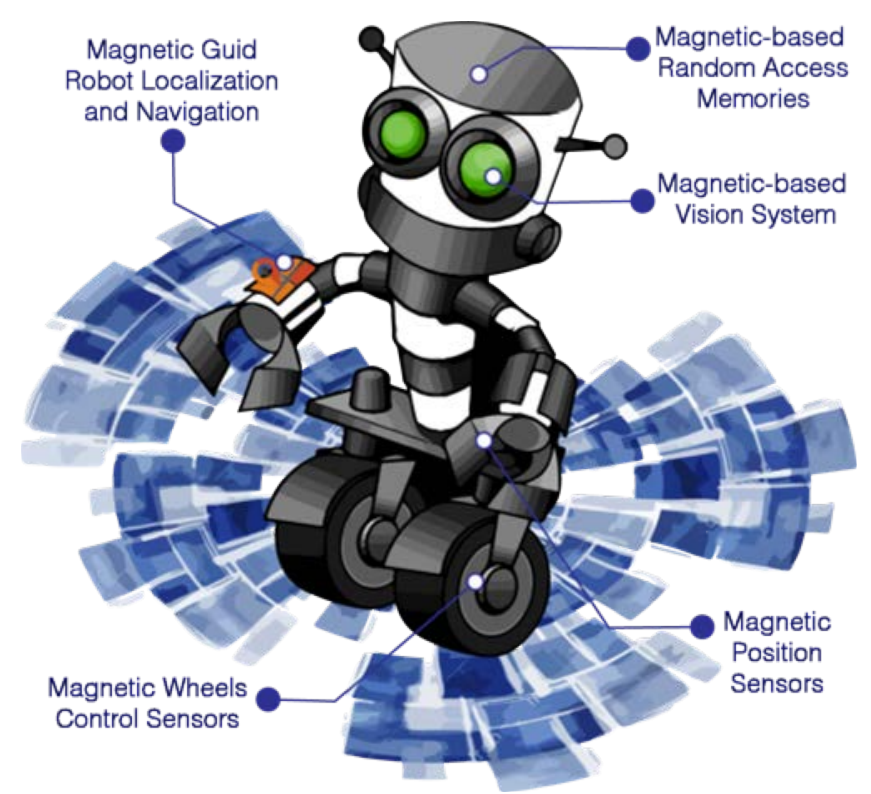

Fig. 1. Different Magnetoelectronics technologies in robotics for precise and natural movement, high reliability and durability and safe operation.

novelty to e-skin concept. This paper also introduces a technology platform that allows designing and fabrication of highly conformable magnetic field sensors. It combines CMOS technology with polymeric foils to achieve flexible sensing elements. The flexible magnetic sensor will be based on $20-\mu \mathrm{m}$ thin Si chip, embedded in about $50-\mu$ m-thick polyimide foils to withstand arbitrary deformations without losing functionality. It is therefore necessary to investigate the effects of all types of stress and consider them in modelling and simulation of bendable devices, which is also the novel aspect of this paper. The new aspects of this work include flexible magnetic microsystem including magnetic sensors and readout CMOS circuit interface, investigations into the stress effects due to bending of chips, and updating device models based on the experimental stress analysis. This paper presents the ultra-thin flexible magnetic sensor and a detailed investigation of mechanical stress effects induced by twisting on the performance of both sub-micron and nanometer scale transistors. It also will focus on the recent progress on the flexible magnetic sensor and predict its application in robotics afterwards.

\section{Magnetic Sensors Methodologies}

\section{A. Hall Effect Sensors}

Hall sensor is the most common type for magnetic sensor. The mature CMOS technology enables the fabrication of high performance magnetic hall sensor in large area and low cost. 


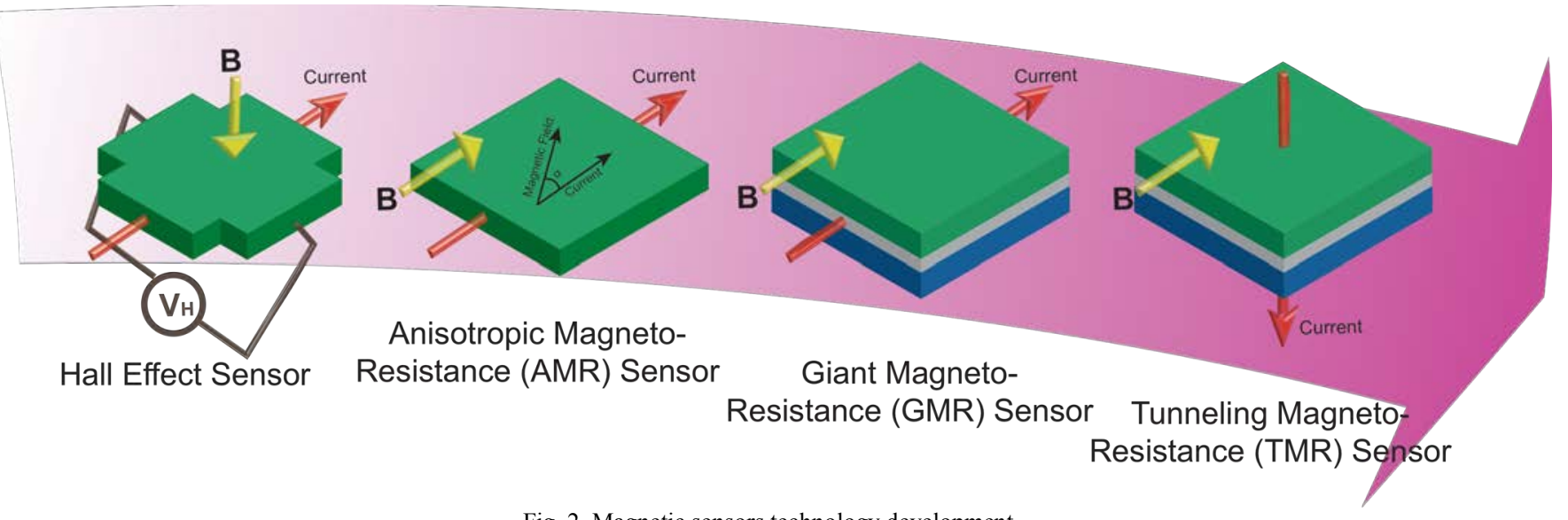

Fig. 2. Magnetic sensors technology development.

Furthermore, integrating the ultra-thin technology with the CMOS based hall sensor, flexibility and conformability can be obtained. Here, we first consider the simplest case on the way towards the flexible Hall sensor. What will happen if we mount the rigid hall sensor onto flexible polymeric substrate? As have been studied [17], if a bulk hall sensor mounted onto a polyimide film, the total structure can sustain a moderate value of bending. In the meantime, the bending will also induce a nonnegligible change to the magnetic sensitivity due to the piezoHall effect. In this case, a compensation of this effect is needed during the measurement. Optimum geometry is predicted theoretically in this case to offer a guide for future study and application. Realizing the Hall sensor fabricating process on top of flexible substrate can be another option towards a flexible magnetic sensor while the selection of proper active material and processing condition are crucial. Recent studies show that initial magnetic film deposition temperature, film thickness and post annealing process for the film are important towards the final device performance on flexible substrate [18]. The flexible substrate itself will result to a change in the film morphology as well as magnetic crystal structure during fabrication process, leading to a non-negligible influence on the magnetic sensing properties of the sensor. However, it is already demonstrated the possibility to fabricate hall sensor with comparable sensitivity as it is on rigid substrate. In the meantime, the flexible Hall sensor can be wrapped around the wrist or pointed by a finger, opening a big window for its future application on bio-monitoring or robotics and autonomous systems control.

\section{B. AMR Sensors}

Studies on AMR sensor can be dated back to long time ago. This type of sensor works under the mechanism of current value dependency on the angle between current direction and magnetic field direction. Compared to most common Hall sensor, the AMR sensor do not suffer from piezo effect, which is favourable for flexible electronics. However, a cross-field error needs to be considered in application [19]. Regarding the real application, a SET/RESET signal is required before each measurement, which is different from all the other magnetic sensor type mentioned above. This unique operating procedure guarantees the accurate sensing ability under different external condition such as large magnetic field or variable temperature.

In the way towards flexible magnetic sensor, the integration of flexible substrate with thin sensor major part is adopted commonly as others. Specifically, technique such as embedding sensing nanowire body inside the porous polymer film [20] and polymer on silicon technology [21] have been used to fabricate the flexible AMR sensor.

\section{GMR Sensors}

If we neglect the tremendous advantage in the large area fabrication in the field of Hall sensor, the GMR sensor almost outperforms the tradition Hall sensor in every aspect, such as sensitivity, temperature stability and power consumption. The mechanism of GMR sensor is from the giant magnetoresistance effect. While ferromagnetic material and non-magnetic material are stacked together, the current passing through the device will have a large dependence on the external magnetic field across the whole structure. In a real device for application, the ferromagnetic and non-magnetic bilayer is repeated multiple times for the purpose of a higher signal response. Up to now, flexibility of the device is introduced by fabricating device on polymer substrate, similar to the case of Hall sensor. The method for depositing active material can be either magnetron sputter directly [22] or printing using the GMR sensitive ink [23]. In the former case, the resistance change can be as high as $57.6 \%$ under $0.4 \mathrm{~T}$, a typical value for $\mathrm{Co} / \mathrm{Cu}$ bilayer system on rigid substrate. While in the latter case, although it is in the same $\mathrm{Co} / \mathrm{Cu}$ bilayer system, due to the performance degradation from the usage of active material ink, the response is around $6 \%$ under $2 \mathrm{~T}$. The flexibility is achieved in both case. The Fig. 2 shows the device under flexed condition.

\section{TMR Sensors}

The TMR refers to the phenomenon that in a magnetic tunnel junction (MTJ) unit, the tunnelling current through the whole structure will have a large dependency on the magnetization direction of the two-ferromagnetic layer separated by a thin insulating layer, as shown in Fig. 1. This effect was discovered in the year 1975 and the relative resistance change at that time was around $14 \%$ at $4.2 \mathrm{~K}$ [24]. With the development of technology, this value has reached to $604 \%$ at room temperature [25] and shows great application potential. A better temperature stability, higher sensitivity and lower power consumption is expected from the TMR sensor, compared with other types. 

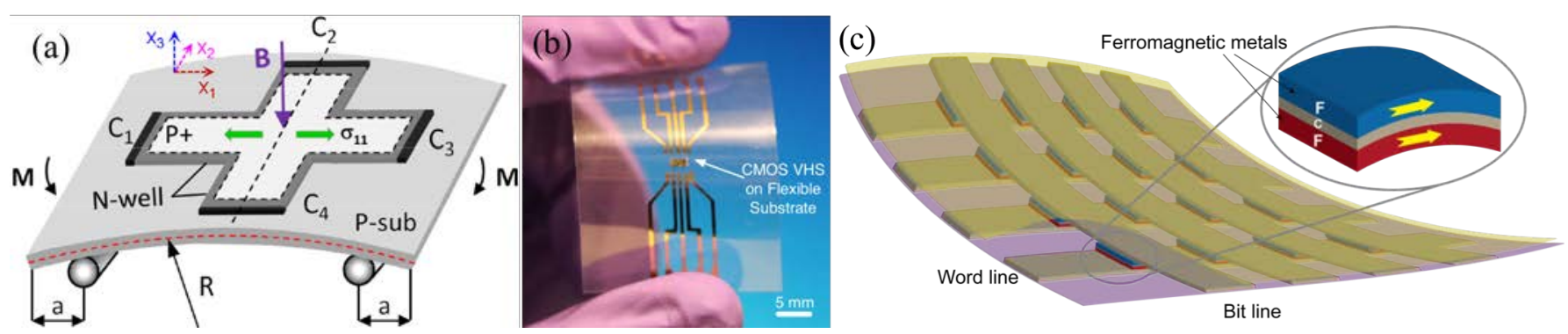

Fig. 3: (a) Horizontal magnetic Hall sensor in a four-point-bending (4PB) setup, (b) the VHS chip mounted on a PVC foil, (c) a schematic representation of random access memory (RAM) that is constructed of arrays of GMR which can be used for large area electronics.

Despite of these advantages, the realization of flexible TMR sensor seems not an easy job. The TMR effect relies on the spin dependent tunnelling process of the device. Due to the sensitive device structure (the insulating tunnel layer is $\sim 2 \mathrm{~nm}$ ), the device deformation may lead to an undesirable result easily, like a direct current short between the two ferromagnetic layers. Therefore, the high surface roughness of conventional flexible substrate rules out the possibility of fabricating TMR sensor directly on top. One possible solution to overcome this barrier, as suggested in a recent report [26], is to use an organic buffer layer on top of the flexible substrate. Atomic force microscope study shows the flexible substrate surface morphology before buffer layer deposition has a peak to peak roughness up to $1 \mu \mathrm{m}$, while after the buffer layer deposition this value decreases to $3 \mathrm{~nm}$. Furthermore, the insulating tunnel barrier remains its insulating electrical property after the deformation test, as confirmed by their conductive atomic force microscope study. Enabled by the addition of buffer layer, large area fabrication of MTJ unit on flexible substrate is possible, which can lead to the potential application of flexible TMR sensor.

\section{FUTURE APPLICATIONS FOR ROBOTICS}

Magnetic sensors are used in a wide range of environments for robotics including humanoid robots, autonomous vehicles and space robotics. With the advent of automation, it becomes increasingly necessary to use sophisticated magnetic sensors as components of a control system in robots. These magnetic sensors were designed to accurately control the machinery through measuring the speed, direction etc. For example, recently in the automotive industry, autonomous vehicles with high-tech sensory systems attracts a lot of attention. The number of magnetic sensors in robots are increasing as per their ambient. Traditional robotic platforms have simple underlying sensory systems which limit their ability to interact with the specific environments they are designed for. Robots often encounter difficulties operating in unstructured and highly congested environments. The sensor's responsiveness and operation is influenced by the environment, for example, the magnetism measurements could vary with temperature, which call for multi-parameter sensor system integration and communication. The wide- operating range of magnetic sensors and the harshness of different environments, call for new designs of sensors and electronics. For example, magnetic sensors by proper material choices and packaging as well as the choice of fabrication technology, can suit various robotic functions. Towards a conformable and adaptive sensory system in robotics, it is important to revisit: (i) materials, (ii) technology, (iii) device design, (iv) packaging and (v) system operation.

Different bending conditions enable different stress effects on silicon, i.e. uniaxial, biaxial, or shear stress effects, which in turn have a different impact on the carriers' mobility of devices. Another important parameter that affects the mobility of carriers is the crystal structure of the silicon substrate and the channel orientation of devices on the substrate. As the first step towards this goal, flexible magnetic sensors (Fig. 3a and Fig. 3b) with simple system integration (Fig. $3 \mathrm{c})$ are illustrated here $[5,17]$. Fig. 3(a) shows the cross-shaped Hall sensor under four-point bending (4PB) setup. The sensor has been simulated using COMSOL Multiphysics as FEM simulator and implemented by Verilog-A in the Cadence environment. The Fig. 2 shows the high-sensitive vertical CMOS-based Hall sensor with readout circuit, which was fabricated using $0.18 \mu \mathrm{m}$ technology and mounted on a flexible substrate. Generally, overcome challenges and advance flexible magnetic sensors, will also allow to expand the scope of research towards modelling the effect of stress and on the performance of magnetic sensors. Fig. 3(c) shows the scheme of bendable microsystem including magnetic sensors blocks on flexible substrate. The main challenges to achieving this are sensor integration, and reliability of electronics on flexible substrates. Overcoming these issues, will lead to technological innovations, which will allow further extend this research towards effective flexible and wearable technology.

With wide range of robotic functions needing variety of magnetic sensor specifications for better control and monitoring, continuous development in field of sensors for robotics is inevitable. The study in new materials, transduction mechanism, novel sensing architecture and processing steps will together contribute to a multi-functional electronic skin and be used in robotic application.

\section{CONCLUSION}

We have presented diverse technologies of magnetic sensors for robotic application. These sensors including Hall, AMR, GMR, and TMR for future applications need to be flexible. In this regard, it's important to consider various aspects of the materials, technology, device design, packaging and system operation for high performance sensing response. The magnetic sensors on flexible substrate 
will open new avenues for a wide range of applications including wearable electronics and interactive electronic-skin for robots and prosthesis.

As for the future work, we will perform simulation and experimental investigations of ultra-thin magnetic sensors during static and dynamic bending, for different in-plane orientations of the sensor and stress application directions. Moreover, the modelling of magnetic sensors under bending stress will be extended for AC investigations. The effects of biaxial and shear stress on the sensor's performance will be also considered.

\section{ACKNOWLEDGEMENT}

This work supported by the Engineering and Physical Sciences Research Council (EPSRC) First Grant (EP/M002519/1) and EPSRC Engineering Fellowship for Growth (EP/M002527/1). We are also thankful from James Watt Nanofabrication Centre (JWNC) and Electronic System Design Centre (ESDC).

\section{REFERENCES}

[1] H. Heidari, U. Gatti, E. Bonizzoni, and F. Maloberti, "Low-Noise Low-Offset Current-Mode Hall Sensors," in IEEE Conf. PhD Research Microelect. Elect. (PRIME), 2013, pp. 325-328.

[2] H. Heidari, E. Bonizzoni, U. Gatti, and F. Maloberti, "A Current-Mode CMOS Integrated Microsystem for Current Spinning Magnetic Hall Sensors," in IEEE Int. Symp. Circuits Systems (ISCAS) 2014, pp. 678-681.

[3] H. Heidari, E. Bonizzoni, U. Gatti, and F. Maloberti, "A 0.18- $\mu \mathrm{m}$ CMOS Current-mode Hall Magnetic Sensor with Very Low Bias Current and High Sensitive Front-End," in Proc. IEEE SENSORS Conf., 2014, pp. 1467-1470.

[4] H. Heidari, E. Bonizzoni, U. Gatti, and F. Maloberti, "Analysis and modeling of four-folded vertical Hall devices in current domain," in IEEE Conf. PhD Research Microelect. Elect. (PRIME), 2014, pp. 1-4.

[5] H. Heidari, N. Wacker, S. Roy, and R. Dahiya, "Towards bendable CMOS magnetic sensors," in IEEE Conf. PhD Research Microelect. Elect. (PRIME), 2015, pp. 314-317.

[6] N. Yogeswaran, W. Dang, W. T. Navaraj, D. Shakthivel, S. Khan, E. O. Polat, et al., "New materials and advances in making electronic skin for interactive robots," Advanced Robotics, vol. 29, pp. 1359-1373, 2015.

[7] R. Dahiya and M. Valle, Robotic tactile sensing: technologies and system: Springer Science \& Business Media, 2013.

[8] A. Vilouras, H. Heidari, S. Gupta, and R. Dahiya, "Modeling of CMOS Devices and Circuits on Flexible Ultrathin Chips," IEEE Trans. Electron Devices, vol. PP, pp. 1-9, 2017.

[9] S. Gupta, H. Heidari, A. Vilouras, L. Lorenzelli, and R. Dahiya, "Device modelling for bendable piezoelectric FET-based touch sensing system," IEEE Transactions on Circuits and Systems I: Regular Papers, vol. 63, pp. 22002208, 2016.

[10] Y. Sonoda, "Applications of magnetometer sensors to observing bio-mechanical movements," IEEE Trans. on Magnetics, vol. 31, pp. 1283-1290, 1995.

[11] S. A. Rahok and K. Ozaki, "Odometry correction with localization based on landmarkless magnetic map for navigation system of indoor mobile robot," in Int. Conf. Autonomous Robots \& Agents, 2009, pp. 572-577.

[12] K. Altun and B. Barshan, "Human Activity Recognition Using Inertial/Magnetic Sensor Units," in Human Behavior Understanding, ed: Springer Berlin Heidelberg, 2010, pp. 38-51.

[13] X. Yun, J. Calusdian, E. R. Bachmann, and R. B. McGhee, "Estimation of Human Foot Motion During Normal Walking Using Inertial and Magnetic Sensor Measurements," IEEE Transactions on Instrumentation and Measurement, vol. 61, pp. 2059-2072, 2012.

[14] W. Wei-Chung, L. Schenato, R. J. Wood, and R. S. Fearing, "Biomimetic sensor suite for flight control of a micromechanical flying insect: design and experimental results," in IEEE Int. Conf. Robotics and Automation, 2003, pp. 1146-1151 vol.1.

[15] S. Weimin, J. Gu, and S. Yanjun, "Proposed wall climbing robot with permanent magnetic tracks for inspecting oil tanks," in IEEE Int. Conf. Mechatronics and Automation, 2005, pp. 2072-2077 Vol. 4.

[16] S. K. Cho, H. Z. Jin, J. M. Lee, and B. Yao, "Teleoperation of a Mobile Robot Using a Force-Reflection Joystick With Sensing Mechanism of Rotating Magnetic Field," IEEE/ASME Transactions on Mechatronics, vol. 15, pp. 17-26, 2010.

[17] H. Heidari, E. Bonizzoni, U. Gatti, F. Maloberti, and R. Dahiya, "CMOS Vertical Hall Magnetic Sensors on Flexible Substrate," IEEE Sensors Journal, vol. 16, pp. 8736-8743, 2016.

[18] M. Melzer, J. I. Mönch, D. Makarov, Y. Zabila, G. S. Cañón Bermúdez, D. Karnaushenko, et al., "Wearable Magnetic Field Sensors for Flexible Electronics," Advanced Materials, vol. 27, pp. 1274-1280, 2015.

[19] P. Ripka and M. Janosek, "Advances in Magnetic Field Sensors," IEEE Sensors J., vol. 10, pp. 1108-1116, 2010.

[20] M. Lindeberg and K. Hjort, "Interconnected nanowire clusters in polyimide for flexible circuits and magnetic sensing applications," Sensors and Actuators A: Physical, vol. 105, pp. 150-161, 7/15/2003.

[21] T. Griesbach, M. C. Wurz, and L. Rissing, "Design, Fabrication, and Testing of a Modular Magnetic Field Microsensor on a Flexible Polymer Foil," IEEE Trans. on Magnetics, vol. 48, pp. 3843-3846, 2012.

[22] M. Melzer, M. Kaltenbrunner, D. Makarov, D. Karnaushenko, D. Karnaushenko, T. Sekitani, et al., "Imperceptible magnetoelectronics," Nature Communications, vol. 6, p. 6080, 2015.

[23] D. Karnaushenko, D. Makarov, C. Yan, R. Streubel, et al., "Printable Giant Magnetoresistive Devices," Advanced Materials, vol. 24, pp. 4518-4522, 2012.

[24] M. Julliere, "Tunneling between ferromagnetic films," Physics Letters A, vol. 54, pp. 225-226, 1975/09/08 1975.

[25] S. Ikeda, J. Hayakawa, Y. Ashizawa, Y. M. Lee, K. Miura, H. Hasegawa, et al., "Tunnel magnetoresistance of 604\% at $300 \mathrm{~K}$ by suppression of $\mathrm{Ta}$ diffusion in $\mathrm{CoFeB} / \mathrm{MgO} / \mathrm{CoFeB}$ pseudo-spin-valves annealed at high temperature," Applied Phys. Let., vol. 93, p. 082508, 2008.

[26] C. Barraud, C. Deranlot, et al., "Magnetoresistance in magnetic tunnel junctions grown on flexible organic substrates," Applied Physics Letters, vol. 96, p. 072502, 2010 . 